\section{Malaria Rapid Diagnostic Test Product Lots Quality Evalua- tion in Ethiopia}

\author{
Sindew Mekasha Feleke*, Markos Sleshi, Gemechu Tadesse \\ and Amha Kebede
}

Ethiopian Public Health Institute (EPHI), Addis Ababa, Ethiopia

\begin{abstract}
As malaria rapidly lead to death, quick and accurate diagnose is important to manage patients. Malaria Rapid Diagnostic Test's (RDTs) has offered extension of diagnosis to remote and poorly resourced areas. However, the qualities vary between different products and batches/lots. Therefore, all production lots must be checked, either pre or post marketing to check lot-lot quality variations and to guarantee end users that RDT result saves lives by guiding the correct treatment. This laboratory evaluation was done in 72 product lots of Plasmodium falciparum (Pf)-only RDT lots tested against positive Pf, Plasmodium vivax $(P v)$ and negative sample panels. The results indicated that $69(95 \%)$ lot RDTs detected the parasite antigen to an acceptable threshold level whereas 3 lots $(4.8 \%)$ showed inadequate sensitivity of the laboratory evaluation. Most product lots passed this laboratory evaluation while some lots showed inadequate sensitivity. Laboratory evaluation of each lot is important to identify and distribute good quality lot RDTs for malaria programme and to ensure the adequate performance of the test.
\end{abstract}

Keywords: Lot-testing; Malaria, RDTs

\section{Introduction}

Malaria is the most important public health problem in Ethiopia hence; the national malaria programme set its goal for control and elimination until 2020 through key strategies such as vector control, case management and environmental management. Therefore, malaria control or elimination strategies require effective patient management, quick and accurate diagnosis [1,2]. In Ethiopia, $P f$ accounts for $60 \%$ and $P v$ for $40 \%$ of malaria cases. Malaria Rapid Diagnostic Tests (RDTs) is a test device that detects parasite antigen in the blood with $>100 \mathrm{p} / \mu \mathrm{l}$ and used for rapid diagnosis and patient management, avoid misdiagnosis of patients who became febrile due to other illness and treat target patients who really have malaria $[3,4]$.

*Corresponding author: Sindew Mekasha Feleke, Ethiopian Public Health Institute (EPHI), Ambo Rd, Addis Ababa, Ethiopia, Tel: +251 941380186; Email: mekashasindeaw@yahoo.com

Citation: Feleke SM, Sleshi M, Tadesse G, Kebede A (2016) Malaria Rapid Diagnostic Test Product Lots Quality Evaluation in Ethiopia. J Infect Non Infect Dis 2: 015.

Received: May 07, 2016; Accepted: May 26, 2016; Published: June 09, 2016
However, the qualities of manufacturing RDTs greatly vary between different products and batches/lots "(each lot is usually identified by a number by the manufacturer and usually consists of 40,000-80,000 tests $[2,5])$ ". For this reason the quality of manufacturers RDT product will be evaluated every two years through product testing programme and WHO releases the result for countries procurement guide. But, since the quality of RDTs between lots of the same product varies due to different manufacturing practice, WHO recommends that all production lots be checked, either pre or post marketing through lot-testing programme [2]. Therefore, this laboratory evaluation of different RDT product lots carried out to assess the quality before or after purchase because lots of most products vary; to convince clinicians, users and regulatory authorities that the tests work and to ensure no damage has occurred during transport to a country (post-purchase testing) [5].

\section{Materials and Methods}

The laboratory evaluation was done in 72 lots of different RDTs (ICT malaria cassette test, Carestart combo, Paracheck Pf, Parascreen pan/Pf and First response Pf/pan). Malaria RDT's detects a parasite antigen of positive bloods with $100 \mathrm{p} / \mu \mathrm{l}$ or more parasite density. However, with this laboratory evaluation $200 \mathrm{p} / \mu \mathrm{l}$ of malaria positive blood used as a minimum threshold value and all tests needs to be positive to pass the evaluation [3]. Each lot- RDTs were tested with positive samples prepared at parasite density of minimum and maximum threshold (200 and 2000 parasite/ $\mu$ ) of $P f$ samples, 200, 500, 2000 parasite/ $\mu \mathrm{l}$ of $P v$ samples and 10 malaria negative panels using WHO protocol $[2,6]$. The blood samples $(10 \mathrm{ml})$ collected from $P f / P v$ malaria positive patients (consented) and the samples prepared to different parasite density $(200,500$ and $2000 \mathrm{p} / \mu \mathrm{l})$ by counting the parasite against white cell using microscopy and diluted with negative blood (prepared from Ab plasma and $\mathrm{O}^{+}$cell). The sample aliquots used for this evaluation was characterized using Polymerase Chain Reaction (PCR) tests for speciation and ELISA test for antigen quantification. The laboratory lot testing was carried out using good samples identified based on the characterization results.

Based on WHO protocol [2], P. falciparum-only RDTs were tested against four different quality control panels and 10 different negative quality control samples. For each of the four quality control $P f$ samples six RDTs were tested at an aliquot of 200 parasites per microliter and one RDT was tested at an aliquot of 2000 parasites per microliter. One RDT was tested with each of the 10 negative quality control samples. $P$. falciparum and pan/combination RDTs were tested against four different $P$. falciparum quality control panels, four $P$. vivax samples and 10 negative quality control samples. For each of the four $P$. falciparum quality control samples, six RDTs were tested at an aliquot of 200 parasites per microliter and one was tested at an aliquot of 2000 parasites per microlitre. For each of the four $P$. vivax quality control samples, six RDTs were tested at an aliquot of 200 parasites per microlitre, and one was tested at an aliquot of 2000 parasites per microlitre. For RDTs failed to detect $P$. vivax at 200 parasites per microlitre; it was re-tested with a $P$. vivax sample diluted at 500 parasites per microliter. One RDT was performed for each of the 10 negative quality control samples. 

015.

This study conducted after getting the ethical approval from Ethiopian Public Health Institute (EPHI) Scientific and Ethical Review Committee (SERC).

\section{Results and Discussion}

The results revealed that $69(95 \%)$ lot RDTs; tested with positive samples of different parasite density $(200 \mathrm{p} / \mu \mathrm{l}$ and $2000 \mathrm{p} / \mu \mathrm{l}$ P. falciparum panels, $200 / 500 \mathrm{p} / \mu \mathrm{l}$ and $2000 \mathrm{p} / \mu \mathrm{l}$ P. vivax panels) and 10 negative samples; detect parasite antigen to an acceptable threshold level whereas 3 lots (4.8\%) nearly equivalent to $120,000-240,000$ tests failed to detect the parasite antigen to an acceptable threshold (Figure 1). Of all the product lot RDTs evaluated, all lots of CareStart Pf/Pan RDTs showed 100\% accuracy and high test band intensity at both high and low parasite density. The lot evaluation results before and after distribution had no variation although the intensity of the reaction was become low with time.

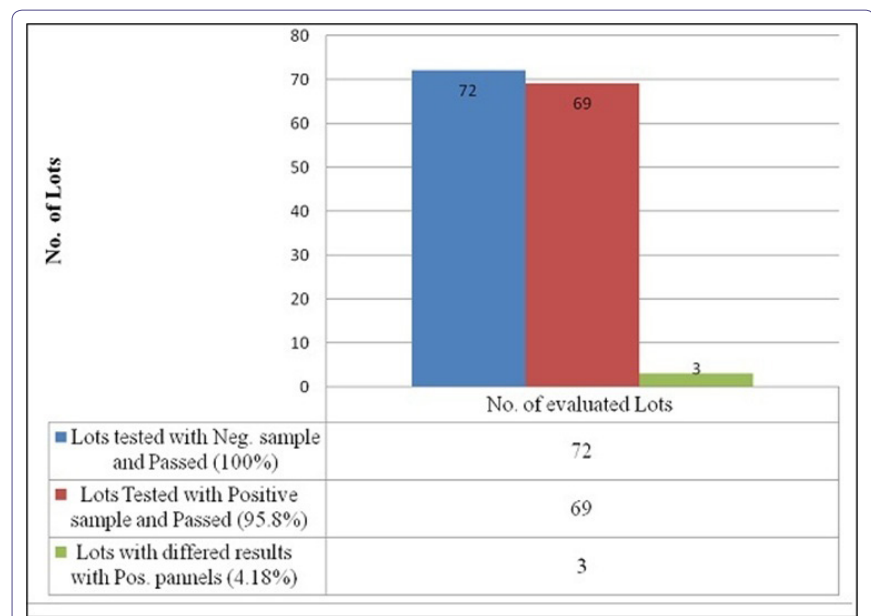

Figure 1: Lot Testing Results of Different Lots Tested with Negative and Positive Analysis.

\section{Conclusion}

The result revealed that most lots passed the laboratory evaluation. Therefore, it is important to ensure continued adequate performance of malaria RDTs lot on delivery and throughout the expected shelf life before and after introducing for national malaria programme. Countries must conduct RDT product lot evaluation to ensure its quality and only confirmed good quality lots should be distributed for the program. This will improve accurate diagnosis and proper treatment of malaria cases.

\section{Acknowledgment}

The authors duly acknowledge the Ethiopian Public Health Institute for financial and technical support as a host organization.

\section{Competing Interests}

The authors declare that there is no any competing interest.

\section{References}

1. Pan American Health organization (2005) Operations Research on the Use of Rapid Diagnostic Tests (RDTs) for Malaria. Pan American Health organization, Washington DC, USA.

2. World Health Organization (2010) Guidelines for the treatment of malaria, $\left(2^{\text {nd }}\right.$ edn), World Health Organization, Geneva, Switzerland.

3. World Health Organization (2011) Methods manual for laboratory based evaluation of malaria rapid diagnostic tests. World Health Organization, Geneva, Switzerland.

4. Bharti PK, Silawat N, Singh PP, Singh MP, Shukla M, et al. (2008) The usefulness of a new rapid diagnostic test, the First Response Malaria Combo (pLDH/HRP2) card test, for malaria diagnosis in the forested belt of central India: Malar J 7: 126.

5. Ethiopian Public Health Institute (2011) Malaria Laboratory Diagnosis External Quality Assessment Scheme Guideline. Ethiopian Public Health Institute, Addis Ababa, Ethiopia.

6. World Health Organization (2010) Malaria rapid diagnostic test performance: summary results of WHO product testing of malaria RDTs: Rounds 1 and 2 (2008-2009): World Health organization on behalf of the Special Programme for Research and Training in Tropical Diseases. World Health Organization, Geneva, Switzerland. 